\title{
Comparison of the inhibition capability of oleanolic acid and betulinic acid towards drug-metabolizing enzymes
}

\author{
Wei Xiao, Meng-Hou Lu
}

Department of Infectious Diseases, Xiangya Hospital of Central South University, Changsha, Hu'nan 410008, China

\begin{abstract}
Background: Human UDP-glucuronosyltransferases (UGTs) are important membrane proteins located in endoplasmic reticulum, and play important roles in metabolism of a variety of endogenous and exogenous compounds.

Aims: To determine the influence of subtle difference in the structure of oleanolic acid and betulinic acid towards the inhibition towards the activity of UGT isoforms.

Methods: In vitro glucuronidation of 4-methylumbelliferone (4-MU) reaction was employed as the probe reaction to determine the inhibition of these two compounds towards UGTs' activity.

Results: The inhibition of capability of oleanolic acid towards UGT1A6 and UGT1A8 were higher than betulinic acid. However, no significant difference was observed for the inhibition of oleanolic acid and betulinic acid towards UGT1A7. Furthermore, concentration-dependent behaviour was determined for the inhibition of oleanolic acid and betulinic acid towards UGT1A6 and UGT1A8. At various concentrations of oleanolic acid and betulinic acid, the inhibition of oleanolic acid towards UGT1A6 and UGT1A8 was higher than betulinic acid.

Conslusion: Given that UGT1A6 and UGT1A8 play key role in the the inhibition of oleanolic acid towards UGT1A6 and UGT1A8 will induce drug-drug interaction and the risk of diseases.

Keywords: UDP-glucuronosyltransferases(UGTs), drug-drug interaction, oleanolic acid, betulinic acid

DOI: http://dx.doi.org/10.4314/ahs.v15i3.40

Cite as: Xiao W, Lu M-H. Comparison of the inbibition capability of oleanolic acid and betulinic acid towards drug-metabolizing ensymes. Afri Health Sci. 2015;15(3):1011-5. doi: http://dx.doi.org/10.4314/abs.v15i3.40
\end{abstract}

\section{Introduction}

Human immunodeficiency virus infection and acquired immune deficiency syndrome (HIV/AIDS) is defined as a disease spectrum of the human immune system caused by infection with human immunodeficiency virus $(\mathrm{HIV})^{1,2}$. Searching the efficient drugs to treat HIV/ AIDS patients is very important and necessary.

Betulinic acid is a naturally occurring pentacyclic triterpenoid, and has been demonstrated to exhibit antiretroviral, antimalarial, and anti-inflammatory biochemical properties. Betulinic acid has been frequently reported to exert anti-tumor activities towards multiple tumor types, including melanoma and breast cancer ${ }^{3,4}$. Oleanolic acid is a naturally occurring triterpenoid isolated from many food and medicinal plants, including Olea europaea (leaves, fruit), Rosa woodsii (leaves), Prosopis glandulosa (leaves and twigs), Phordendron juniperinum (whole plant), Syzygium claviflorum (leaves), Hyptis capitata (whole plant), Mirabilis jalapa and Ternstromia gymnanthera (aerial part) ${ }^{5}$. Oleanolic acid has very similar structure with betulinic acid (Figure 1).

Betulinic acid and oleanolic acid are also the potential drug candidates for the treatment of HIV/AIDS patients ${ }^{6}$.

\section{Corresponding author: \\ Meng-Hou Lu, \\ Department of Infectious Diseases, \\ Xiangya Hospital of Central South University, \\ Changsha, Hu'nan 410008, China \\ E-mail: lumenghouxiangya@163.com}




\section{Figure 1}

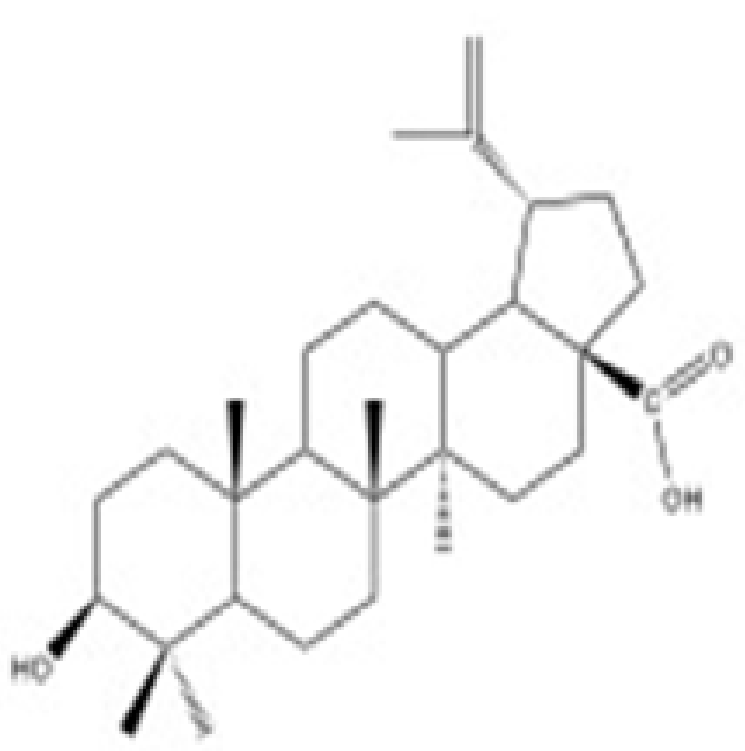

Betulinic acid

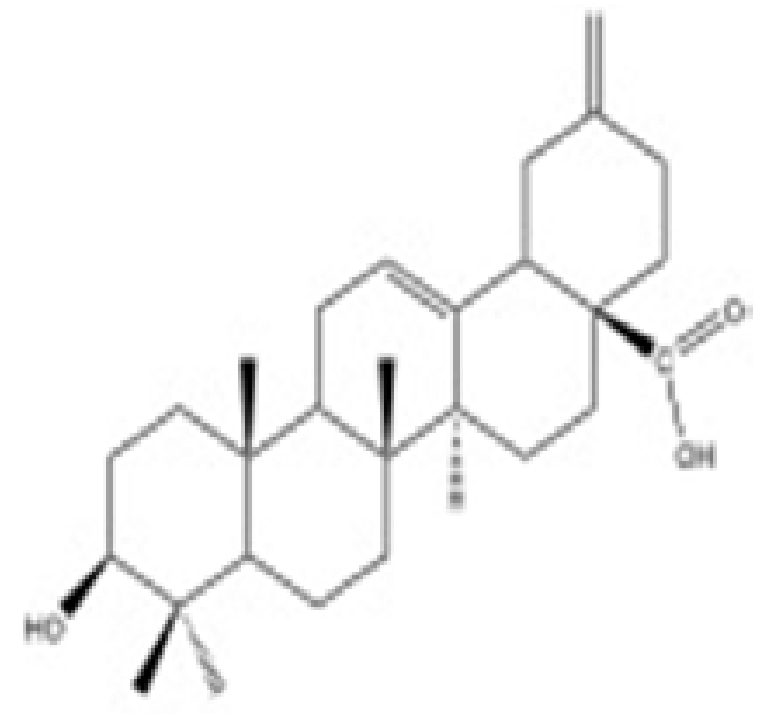

Oleanolic acid
The present study aims to investigate the inhibition capability of these two compounds towards the activity of drug-metabolizing enzymes UDP-glucuronosyltransferases (UGTs) 1A6, 1A7, and 1A8.

\section{Materials and methods}

\section{Chemicals and reagents}

4-Methylumbelliferone(4-MU),4-methylumbelliferone-b-D-glucuronide(4-MUG), Tris-HCl,7-hydroxycoumarin, and uridine-diphospho-glucuronic acid trisodium salt (UDPGA) were purchased from Sigma-Aldrich. Recombinant UDP-glucuronosyltransferase (UGT) isoforms were obtained from BD Gentest Corp. (Woburn, MA). All other reagents were of HPLC grade or of the highest grade commercially available.

Investigation of the inhibition of UGTs' activity by oleanolic acid and betulinic acid

For UGT supersomes-catalyzed in 4-MU glucuronida- tion probe reactions, the incubation system $(200 \mathrm{uL})$ is consisted of $0.05 \mathrm{mg} / \mathrm{ml} \mathrm{UGT}$ supersomes, $5 \mathrm{mM}$ UDPGA co-factor, $5 \mathrm{mM}$ of $\mathrm{MgCl} 2,50 \mathrm{mM}$ Tris- $\mathrm{HCl}$ $(\mathrm{pH}=7.4)$, and $4-\mathrm{MU}$ in the absence or presence of different concentration of oleanolic acid and betulinic acid. The concentration of used protein and the incubation time can ensure the linear reaction. The concentration of 4-MU was equal to the $\mathrm{Km}$ or S50 values for each UGT isoform. The analytical conditions have been described in the previous literatures ${ }^{7,8}$.

\section{Statistical method}

The statistical difference was performed using twotailed student t-test.

\section{Results}

The inhibition screening of oleanolic acid and betulinic acid towards three important UGT isoforms UGT1A6, UGT1A7, and UGT1A8 was firstly investigated, and the results were given in Figure 2. 
Figure 2 Initial screening the inhibition capability of oleanolic acid and betulinic acid towards the activity of UGT1A6, UGT1A7, and UGT1A8. $100 \mathrm{uM}$ of oleanolic acid and betulinic acid were used, and the statistical difference was given as $* * * \mathbf{p}<0.001$.

\section{Figure 2}
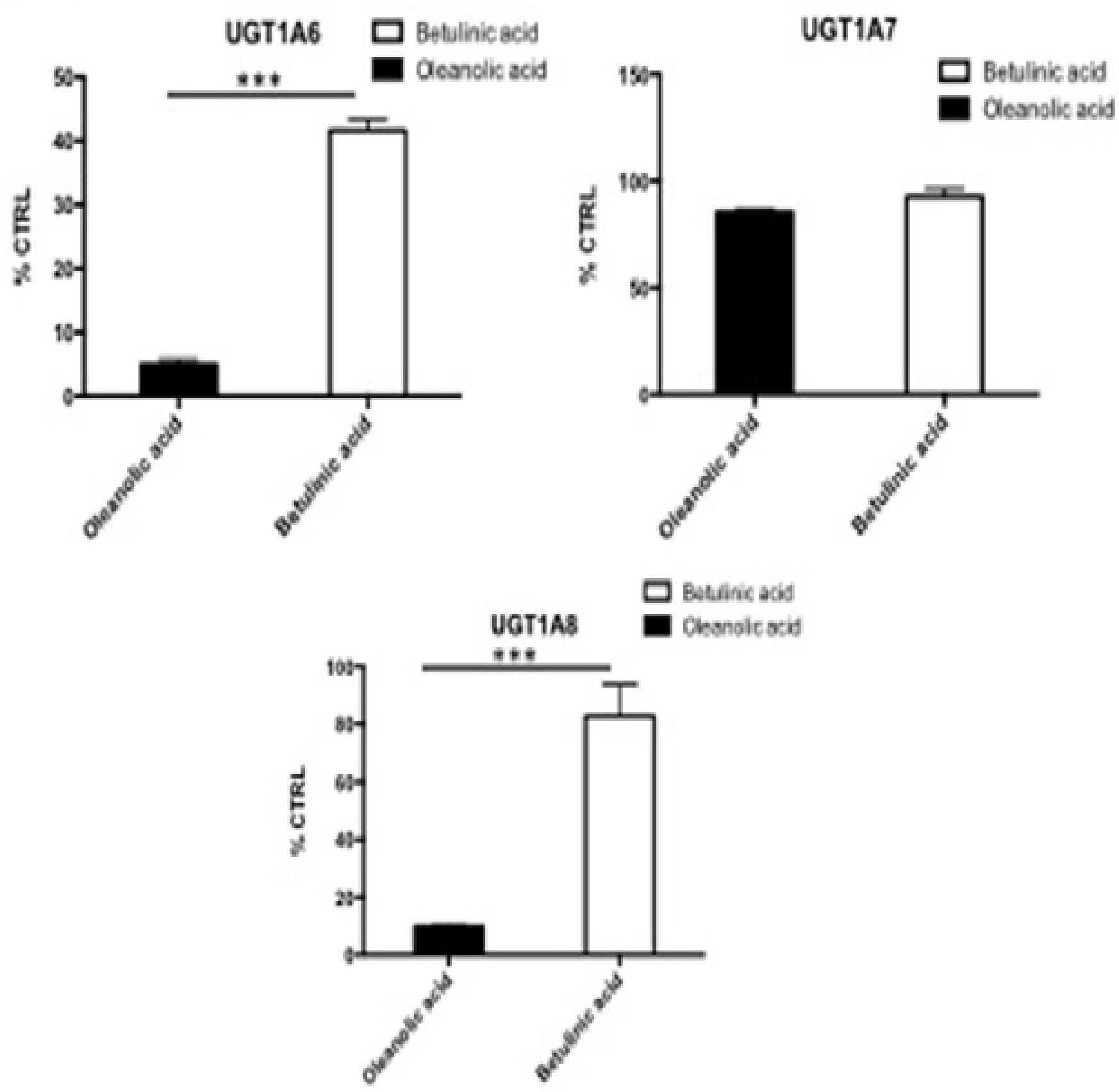

The inhibition of capability of oleanolic acid towards UGT1A6 and UGT1A8 were higher than betulinic acid. However, no significant difference was observed for the inhibition of oleanolic acid and betulinic acid towards UGT1A8. Furthermore, concentration-dependent behaviour was determined for the inhibition of oleanolic acid and betulinic acid towards UGT1A6 and UGT1A8 (Figure 3).

At various concentrations of oleanolic acid and betulinic acid, the inhibition of oleanolic acid towards UGT1A6 and UGT1A8 was higher than betulinic acid. 


\section{Figure 3}

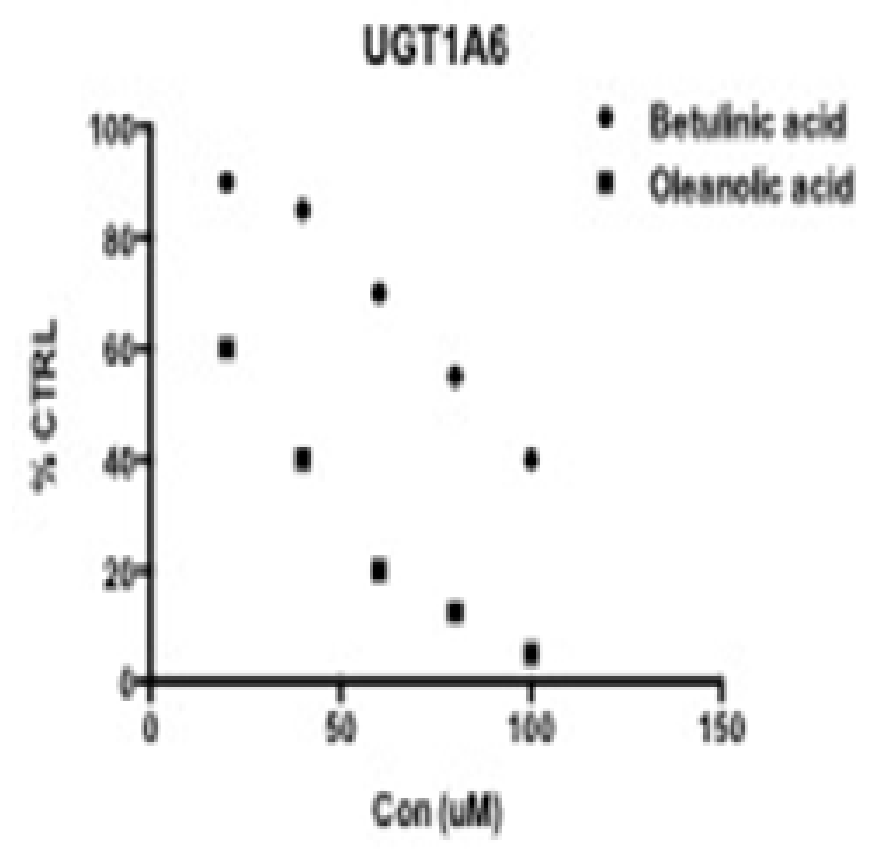

\section{Discussion}

Human UDP-glucuronosyltransferases (UGTs) are important membrane proteins located in endoplasmic reticulum, and play important roles in metabolism of a variety of endogenous and exogenous compounds. Many UGTs-inhibition based adverse effects cases have been reported. For example, indinavir, an HIV therapeutic drug, can significantly induce the elevation of unconjugated bilirubin in serum through inhibition of UGT1A1-mediated bilirubin glucuronidation ${ }^{10}$.

Many compounds have been reported to exert inhibition potential towards UGT isoforms, such as warfa$\operatorname{rin}^{11}$ and $\operatorname{arbidol}^{12}$. The present study showed that subtle difference for the structure of oleanolic acid and betulinic acid will result in the significant difference of these two compounds' inhibition towards UGT1A6 and UGT1A8. Besides the high contribution of these two UGT isoforms towards the metabolism of xenobiotics, high correlation between the activity of these two UGT isoforms and cancers was reported. For example,

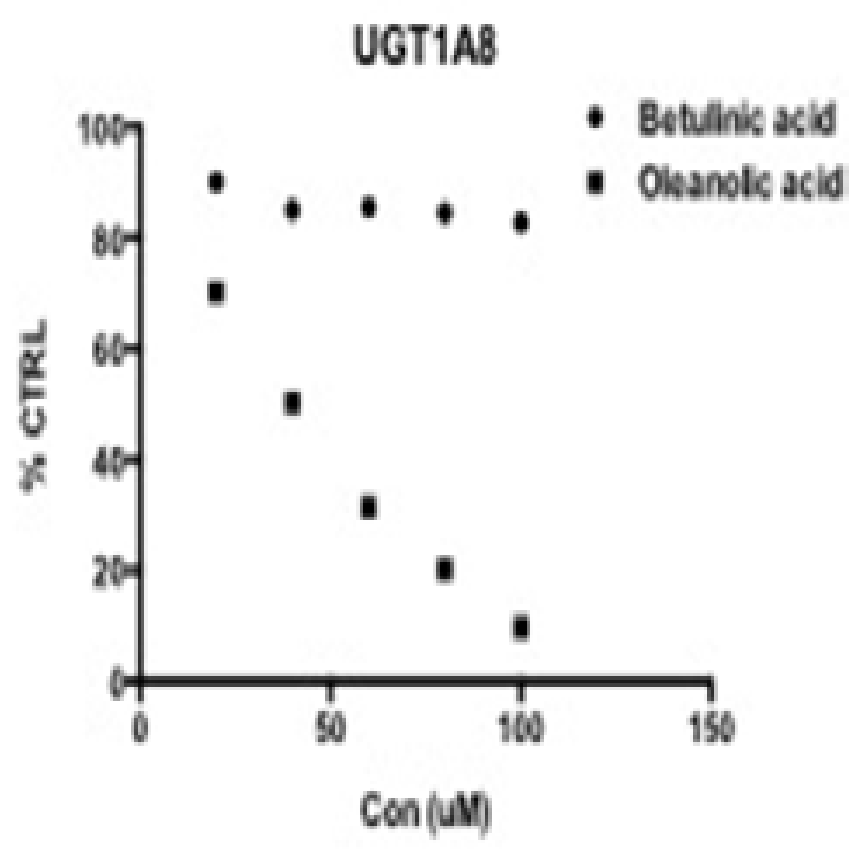

UGT1A6_19_GG genotype has been reported to be a breast cancer risk factor ${ }^{13}$. The lower activity of UGT1A8 will increase the risk of esophageal cancer risk ${ }^{14}$. Therefore, the inhibition of oleanolic acid towards UGT1A6 and UGT1A8 will induce drug-drug interaction and the risk of diseases.

\section{References}

1. Williamson RT, Wondergem P, Amenyah R. (2014) Using a Reporting System to Protect the Human Rights of People Living with HIV and Key Populations: A Conceptual Framework. Health Hum Rights 2014; 16(1): E148-E156.

2. Davey NE, Satagopam VP, Santiago-Mozos S, Villacorta-Martin C, Bharat TA, Schneider R, Briggs JA. The HIV Mutation Browser: A Resource for Human Immunodeficiency Virus Mutagenesis and Polymorphism Data. PLoS Comput Biol 2014;10(12):e1003951.

3. Park SY, Kim HJ, Kim KR, Lee SK, Lee CK, Park $\mathrm{KK}$, et al. Betulinic acid, a bioactive pentacyclic triterpenoid, inhibits skeletal-related events induced by 
breast cancer bone metastases and treatment. Toxicol Appl Pharmacol 2014; 275(2): 152-162.

4. Hung HY, Nakagawa-Goto $K$, Tokuda $H$, lida A, Suzuki N, Bori ID, et al. A-ring modified betulinic acid derivatives as potent cancer preventive agents. Bioorg Med Chem Lett 24(3): 1005-1008.

5. Zhao X, Liu M, Li D. Oleanolic acid suppresses the proliferation of lung carcinoma cells by miR-122/ Cyclin G1/MEF2D axis. Mol Cell Biochem 2014; doi: 10.1007/s11010-014-2228-7.

6. Dang Z, Ho P, Zhu L, Qian K, Lee KH, Huang L, et al. New betulinic acid derivatives for bevirimat-resistant human immunodeficiency virus type-1.J Med Chem 56(5): 2029-2037.

7. Fang ZZ, Cao YF, Hu CM, Hong M, Sun XY, Ge $\mathrm{GB}$, et al. Structure-inhibition relationship of ginsenosides towards UDP-glucuronosyltransferases (UGTs). Toxicol Appl Pharmacol 267(2): 149-154.

8. Liu XY, Fang ZZ, Dong PP, Shi XH, Teng YJ, Sun XY. Tacrolimus strongly inhibits multiple human UDP-glucuronosyltransferase (UGT) isoforms. Pharmazie 2012; 67(9): 804 PubMed -808.

9. Biao C, Caron S, Dallaire-Theroux A, Barbier O. Nu- clear receptors and endobiotics glucuronidation: the good, the bad, and the UGT. Drug Metab Rev 2013; 45(1): 34 PubMed -47.

10. Zucker SD, Qin X, Rouster SD, Yu F, Green RM, Keshavan P, et al. Mechanism of indinavir-induced hyperbilirubinemia. Proc Natl Acad Sci USA 2001; 98(22): 12671-12676.

11. Sun H, Zhang T, Wu Z, Wu B. Warfarin is an effective modifier of multiple UDP-glucuronosyltransferase enzymes: evaluation of its potential to alter the pharmacokinetics of zidovudine. J Pharm Sci 2014; doi: 10.1002/jps.24250.

12. Liu X, Huang T, Chen JX, Zeng J, Fan XR, Zhu $\mathrm{X}$, et al. Arbidol exhibits strong inhibition towards UDP-glucuronosyltransferase (UGT) 1A9 and $2 \mathrm{~B} 7$. Pharmazie 2013; 68(12): 945 PubMed -950.

13. Justenhoven $\mathrm{C}$, Obazee $\mathrm{O}$, Winter $\mathrm{S}$, Rabstein $\mathrm{S}$, Lotz A, Harth V, et al. The UGT1A6_19_GG genotype is a breast cancer risk factor. Front Genet 4:104.

14. Dura P, Salomon J, Te Morsche RH, Roelofs HM, Kristinsson JO, Wobbes T, et al. High enzyme activity UGT1A1 or low activity UGT1A8 and UGT2B4 genotypes increase esophageal cancer risk. Int J Oncol 2012; 40(6): 1789 PubMed -1796. 